\title{
Stability and Instability of Certain Foliations of 4-Manifolds by Closed Orientable Surfaces
}

By

\author{
Kazuhiko FukUI*
}

\section{§0. Introduction}

Let $\mathrm{Fol}_{q}(M)$ denote the set of codimension $q C^{\infty}$-foliations of a closed $m$-manifold $M$. Fol $_{q}(M)$ carries a natural weak $C^{r}$-topology $(0 \leq r \leq \infty)$, which is described in [7]. We denote this space by $\operatorname{Fol}_{q}^{r}(M)$. We say a foliation $F$ is $C^{r}$-stable if there exists a neighborhood $V$ of $F$ in $\operatorname{Fol}_{q}^{r}(M)$ such that every foliation in $V$ has a compact leaf. We say $F$ is $C^{r}$-unstable if not. A foliation in a small neighborhood of $F$ in $\operatorname{Fol}_{q}^{r}(M)$ is said to be a $C^{r}$-perturbation of $F$. It seems to be of interest to determine if $F$ is $C^{r}$-stable or not. Let $L$ be a compact leaf of $F$. Langevin-Rosenberg [8] showed, generalizing the Reeb stability theorem [11], that if $H^{1}(L ; \mathbb{R})=0$, then $F$ is $C^{1}$-stable. Let $\pi_{1}(L) \rightarrow G L(q, \mathbb{R})$ be the action determined from the linear holonomy of $L$, where $q$ is the codimension of $F$. Then generalizing the results of Hirsch [7] and Thurston [16], Stowe [15] showed that if the cohomology group $H^{1}\left(\pi_{1}(L)\right.$; $\mathbb{R})$ is trivial, then $F$ is $C^{1}$-stable. On the other hand, let $F$ be the foliation of an orientable $S^{1}$-bundle over a closed surface $B$ by fibres. Seifert [13] showed that $F$ is $C^{0}$-stable if $\chi(B) \neq 0$, where $\chi(B)$ is the euler characteristic of $B$. The result was generalized by Fuller [6] to orientable circle bundles over arbitrary closed manifolds $B$ with $\chi(B) \neq 0$. Langevin-Rosenberg [9] considered a fibration $\pi: M \rightarrow B$ with fibre $L$ and showed that the foliation of $M$ by fibres is $C^{0}$-stable provided that 1) $\left.\pi_{1}(L) \cong \mathbb{Z}, 2\right) B$ is a closed surface with $\chi(B) \neq 0$ and 3$) \pi_{1}(B)$ acts trivially on $\pi_{1}(L)$. The author [4] generalized the above result to compact codimension two foliations. Plante [10] classified all foliations of closed 3-manifolds by closed orientable surfaces into stable or unstable foliations. The author [5] classified all foliations of closed 3-manifolds by circles into stable or unstable foliations.

Communicated by N. Shimada, June 6, 1986. Revised August 2, 1986.

* Department of Mathematics, Kyoto Sangyo University, Kyoto 603, Japan. 
We study here the case $M$ is a closed 4-manifold and $F$ is a foliation of $M$ by closed orientable surfaces. Our main results are as follows. See $\S 1$ for definitions.

Theorem A (Theorem 8). Let $F$ be a foliation with all leaves tori and only reflection leaves as singular leaves. Then we can regard a union of reflection leaves as $T^{2} \times[0,1] / h$, where $h$ is a diffeomorphism of $T^{2}$. If the induced automorphism $h_{*}: H_{1}\left(T^{2} ; \mathbb{Z}\right) \rightarrow H_{1}\left(T^{2} ; \mathbb{Z}\right)$ is equal to $\left(\begin{array}{rr}-1 & 0 \\ 0 & -1\end{array}\right)$, then $F$ is $C^{1}$-stable.

Theorem $\mathbb{B}$ (Theorem 17)。 Let $F$ be a foliation of $M$ without singular leaves. Then $F$ is $C^{r}$-unstable $(r \geq 0)$ if one of the following is satisfied;

(1) $M / F$ is homeomorphic to the 2-sphere and the genus of a generic leaf $\geq 2$, (2) $M / F$ is homeomorphic to the projective plane and the genus of a generic leaf $\geq 4$,

(3) $M / F$ is neither homeomorphic to the 2-sphere nor the projective plane and the genus of a generic leaf $\geq 6$.

Theorem $\mathbb{C}$ (Theorem 25)。 Let $F$ be a foliation of $M$ with generic leaf of genus $g$ and $B=M / F$ be the leaf space. Suppose $F$ has $m$ rotation leaves with holonomy groups $\mathbb{Z}_{k_{i}}(i=1,2, \cdots, m)$ and $m_{j}$ dihedral leaves with holonomy groups $\mathbb{D}_{l_{j, k}}\left(k=1,2, \cdots, m_{j}\right)$ which correspond to points of $\partial_{j} B$ for each $j\left(1 \leq j \leq n^{\prime}\right)$. If $g \geq \max \left(3 \max \left(k_{i} ; 1 \leq i \leq m\right)+1,8 \max \left(l_{j, k} ; 1 \leq j \leq n^{\prime}, 1 \leq k \leq m_{j}\right)+1,7 \varepsilon\right)$, then $F$ is $C^{r}$-unstable $(r \geq 0)$, where $\varepsilon=0$ or 1 and $F$ has no reflection leaves if and only if $\varepsilon=0$.

The paper is organized as follows. In $\S 1$, we recall the (local) structure of compact codimension two foliations and prepare some definitions and notations. In $\S 2$, we discuss about foliations with all leaves tori and prove Theorem A. In $\S 3$, we discuss about foliations with generic leaf of genus $\geq 2$ and no singular leaves and prove Theorem B. In $\S 4$, we discuss about foliations with generic leaf of genus $\geq 2$ and singular leaves and prove Theorem $\mathrm{C}$. All foliations we consider here are smooth of class $C^{\infty}$ and of codimension two.

\section{$\S 1$. Compact Foliations and Singular Leaves}

Let $M$ be a closed manifold and $F$ a compact foliation of codimension two. By the results of Epstein [2] and Edwards-Millett-Sullivan [1], we have a nice picture of the local behavior of $F$ as follows.

Proposition 1 (Epstein [3]). There is a generic leaf $L_{0}$ with property that 
there is an open dense saturated subset of $M$, where all leaves have trivial holonomy and are diffeomorphic to $L_{0}$. Given a leaf $L$, we can describe a neighborhood $U(L)$ of $L$, together with the foliation on the neighborhood as follows. There is a finite subgroup $G(L)$ of $O(2)$ such that $G(L)$ acts freely on $L_{0}$ on the right and $L_{0} / G(L) \cong L$. Let $D^{2}$ be the unit disk. We foliaie $L_{0} \times D^{2}$ with leaves of the form $L_{0} \times\{p t\}$. This foliation is preserved by the diagonal action of $G(L)$, defined by $g(x, y)=\left(x \cdot g^{-1}, g \circ y\right)$ for $g \in G(L), x \in L_{0}$ and $y \in D^{2}$, where $G(L)$ acts linearly on $D^{2}$. So we have a foliation induced on $U=L_{0} \times D_{(G L)}^{2}$. The leaf corresponding to $y=0$ is $L_{0} / G(L)$. Then there is a $C^{\infty}$-imbedding $\varphi: U \rightarrow M$ with $\varphi(U)=U(L)$, which preserves leaves and $\varphi\left(L_{0} / G(L)\right)=L$.

Remark 2. $U(L)$ can be considered to be the total space of a normal disk bundle of $L$ in $M$ with structure group $G(L)$.

Since $G(L)$ is a finite subgroup of $O(2), G(L)$ is isomorphic to a rotation group $\mathbb{Z}_{k}(k>1)$, a dihedral group $\mathbb{D}_{l}(l>1)$ which consists of $l$ rotations and $\ell$ reflections or a group $\mathbb{D}$ consisting of only one reflection, which is called a reflection group.

Defingition 3. A leaf $L$ is singular if $G(L)$ is not trivial. The order of $G(L)$ is called the order of holonomy of $L$. We say such an $L$ is a rotation leaf, a reflection leaf or a dihedral leaf if $G(L)$ is isomorphic to $\mathbb{Z}_{k}(k>1), \mathbb{D}$ or $\mathbb{D}_{l}(\ell>1)$ respectively.

Let $B=M / F$ be the leaf space. $B$ is a compact $V$-manifold of dimension two and is also a topological manifold. The quotient map $\pi: M \rightarrow B$ is a $V$-bundle (see Satake [12] for definitions). Since $M$ is compact, there are finitely many rotation leaves and dihedral leaves in $F$. Dihedral and reflection leaves correspond to the boundary points of $B$. Let $n\left(=n^{\prime}+n^{\prime \prime}\right)$ be the number of boundary components of $B$. We let $L_{i}(i=1,2, \cdots, m)$ be all rotation leaves with holonomy groups $\mathbb{Z}_{k_{i}}$ and $L_{j, k}\left(j=1,2, \cdots, n^{\prime} ; k=1,2, \cdots, m_{j}\right)$ all dihedral leaves with holonomy groups $\mathbb{D}_{l_{j, k}}$ respectively such that $L_{j, k}$ $\left(k=1,2, \cdots, m_{j}\right)$ correspond to points in the $j$-th boundary $\partial_{j} B$ of $B\left(1 \leq j \leq n^{\prime}\right)$. All points in other boundaries $\partial_{n^{\prime}+j} B\left(1 \leq j \leq n^{\prime \prime}\right)$ of $B$ correspond to reflection leaves. Choose saturated neighborhoods $U\left(L_{i}\right)$ as in Proposition 1 to be disjoint and take saturated neighborhoods $U^{\prime}\left(L_{i}\right)$ such that $U^{\prime}\left(L_{i}\right) \subset \stackrel{\circ}{U}\left(L_{i}\right)$, where $\stackrel{\circ}{U}$ denotes the interior of $U$. Let $V_{i}=\pi\left(U\left(L_{i}\right)\right)$; and $V_{i}^{\prime}=\pi\left(U^{\prime}\left(L_{i}\right)\right)$. Let $B_{0}=B-\bigcup_{i=1}^{m} \stackrel{\circ}{V}_{i}^{\prime}, B_{1}=B_{0}-\partial B$ and $M_{1}=\pi^{-1}\left(B_{1}\right)$. The restricted map $\pi$ : $M_{1} \rightarrow B_{1}$ is a fibre bundle with generic leaf $L$ as fibre. We now assume that 
$M$ is a closed 4-manifold and $F$ is a foliation of $M$ by closed orientable surfaces of genus $\geq 2$. Then the bundle $\pi: M_{1} \rightarrow B_{1}$ is represented as follows. Let $B_{0}$ be a compact surface of genus $h$ with $m+n$ boundaries. First we consider the case $B$ is orientable. Take simple closed curves $c_{i}(i=1,2, \cdots, 2 h)$ and $\operatorname{arcs} d_{j}(j=1,2, \cdots, m+n)$ on $B_{0}$ such that 1) $c_{i}$ and $c_{i+1}$ intersect at points $\left.p_{i}(i=1,2, \cdots, 2 h-1), 2\right) d_{j}(j=1,2, \cdots, m)$ join points $p_{2 h+j-1}$ of $c_{2 h}$ and $\partial V_{j}^{\prime}$ respectively and $d_{m+j}(j=1, \cdots, n)$ join points $p_{2 h+m+j-1}$ of $c_{2 h}$ and points $q_{j}$ of $\partial_{j} B$, where $q_{j}=\pi\left(L_{j, 1}\right)$ for $j=1, \cdots, n^{\prime}$, and 4) cutting off $B_{0}$ along $c_{i}(i=1$, $\cdots, 2 h)$ and $d_{j}(j=1, \cdots, m+n)$ yields a compact manifold $B_{2}$ which is homeomorphic to a disk (see Fig. 1). $c_{1}$ is separated to an arc $c_{1}^{\prime}$ by $c_{2}, c_{i}(i=2, \cdots$,

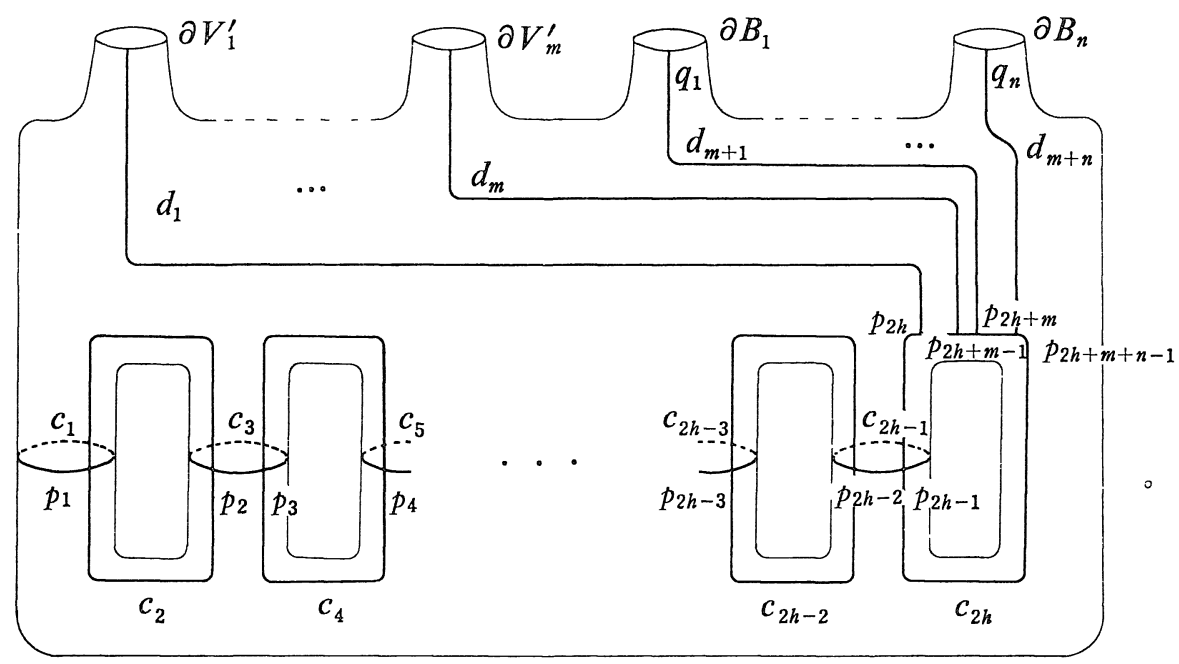

Figure 1

$2 h-1)$ are separated to two $\operatorname{arcs} c_{i 1}, c_{i, 2}$ by $c_{i-1}$ and $c_{i+1}$, and $c_{2 h}$ is separated to $m+n+1$ arcs $c_{2 h, j}(j=1, \cdots, m+n+1)$ by $c_{2 h-1}$ and $d_{j}(j=1, \cdots, m+n)$. Cutting off $B_{1}$ along $c_{i}$ and $d_{j}$ yields a subset $B_{3}$ of $B_{2}$ whose interior is homeomorphic to an open disk. Then we obtain $M_{1}$ by making the following identifications in $L \times B_{3}$ as follows;

$(x, y) \sim\left(\varphi_{1}(y)(x), y\right)$ for $x \in L, y \in c_{1}^{\prime},(x, y) \sim\left(\varphi_{i j}(y)(x), y\right)$ for $x \in L, y \in c_{i j}$ $(i=2, \cdots, 2 h-1 ; j=1,2),(x, y) \sim\left(\varphi_{2 h, j}(y)(x), y\right)$ for $x \in L, y \in c_{2 h, j}(j=1, \cdots$, $m+n+1)$ and $(x, y) \sim\left(\psi_{k}(y)(x), y\right)$ for $x \in L, y \in d_{k}(k=1, \cdots, m+n)$, where $\varphi_{1}: c_{1}^{\prime} \times L \rightarrow L, \varphi_{i j}: c_{i j} \times L \rightarrow L \varphi_{2 h, j}: c_{2 h, j} \times L \rightarrow L$ and $\psi_{k}: d_{k} \times L \rightarrow L$ are smooth maps such that $\varphi_{1}(y), \varphi_{i j}(y), \varphi_{2 h, j}(y)$ and $\psi_{k}(y)$ for $y \in c_{1}^{\prime}, c_{i j}, c_{2 h, j}$ and $d_{k}$ are diffeomorphisms of $L$ respectively. We may assume that $\varphi_{1}(y), \varphi_{i j}(y), \varphi_{2 h, j}(y)$ and $\psi_{k}(y)$ are constant diffeomorphisms on neighborhoods of the boundaries 
of $c_{1}^{\prime}, c_{i j}, c_{2 h, j}$ and $d_{k}$ respectively.

Next we consider the non-orientable case. Let $B_{0}^{\prime}$ be a closed surface obtained by pasting disks to $B_{0}$ along the boundaries. $B_{0}^{\prime}$ is homeomorphic to $\sum_{h_{1}} \# P^{2}$ or $\sum_{h_{1}} \# K^{2}$ according to $h=2 h_{1}+1$ or $2 h_{1}+2$, where $\sum_{h_{1}}$ is an orientable surface of genus $h_{1}, P^{2}$ is the projective plane and $K^{2}$ is the Klein bottle. We can identify $B_{0}$ with $\sum_{h_{1}} \# P^{2}-\bigcup_{i=1}^{m+n} D_{i}^{2}$ or $\sum_{h_{1}} \# K^{2}-\bigcup_{i=1}^{m+n} D_{i}^{2}$.

Case of $h=2 h_{1}+1$. Take simple closed curves $c_{i}(i=1,3,4, \cdots, h+1)$ and $\operatorname{arcs} c_{2}, d_{j}(j=1,2, \cdots, m+n)$ on $B_{0}$ such that 1) $c_{i}$ and $c_{i+1}$ intersect at points $\left.p_{i}(i=3,4, \cdots, h), 2\right) c_{1}$ generates the fundamental group of $\left.P^{2}, 3\right) c_{2}$ joins a point $p_{1}$ of $c_{1}$ and a point $p_{2}$ of $\left.c_{3}, 4\right) d_{j}(j=1, \cdots, m)$ join points $p_{h+j}$ of $c_{h+1}$ and $\partial V_{j}^{\prime}, d_{m+j}(j=1, \cdots, n)$ join points $p_{h+m+j}$ of $c_{h+1}$ and points $q_{j}$ of $\partial_{j} B$, where $q_{j}=\pi\left(L_{j, 1}\right)$ for $j=1, \cdots, n^{\prime}$ and 5) cutting off $B_{0}$ along $c_{i}(i=1, \cdots$, $h+1)$ and $d_{j}(j=1, \cdots, m+n)$ yields a compact topological manifold $B_{2}$ which is homeomorphic to a disk.

Case of $h=2 h_{1}+2$. Take simple closed curves $c_{1}, c_{2}$ on $B_{0}$ instead of $c_{1}, c_{2}$ in the case of $h=2 h_{1}+1$ as follows: their homotopy classes $\left\{c_{1}\right\}$ and $\left\{c_{2}\right\}$ are generators of the fundamental group of $K^{2}$ with the relation $\left\{c_{1}\right\} \cdot\left\{c_{2}\right\}$ $=\left\{c_{2}\right\}^{-1} \cdot\left\{c_{1}\right\}$ and $c_{1}$ and $c_{2}$ intersect at $p_{1}$. Take other simple closed curves $c_{i}(i=3,4, \cdots, h+1)$ and $\operatorname{arcs} d_{j}(j=1,2, \cdots, m+n)$ on $B_{0}$ in the same way as in the case of $h=2 h_{1}+1$. Note that $c_{2}$ and $c_{3}$ intersect at a point $p_{2}$. $c_{1}$ is separated to an arc $c_{1}^{\prime}$ by $c_{2}, c_{i}\left(i=3,4, \cdots, 2 h_{1}-1\right)$ are separated to two arcs $c_{i, 1}, c_{i, 2}$ by $c_{i-1}$ and $c_{i+1}$, and $c_{2 h}$ is separated to $m+n \operatorname{arcs} c_{2 h, j}(j=1,2, \cdots$, $m+n)$ by $c_{2 h-1}$ and $d_{j}(j=1,2, \cdots, m+n)$. In the case of $h=2 h_{1}+2, c_{2}$ is separated to two $\operatorname{arcs} c_{2,1}, c_{2,2}$ by $c_{1}$ and $c_{3}$. The rest is similar to the orientable case.

Definition 4. Let $Y$ be a subset of a manifold $X$. We say a diffeomorphism $f: X \rightarrow X$ satisfies the property $P(Y, r)$ if 1$) f$ is sufficiently $C^{\prime \prime}$-close to $1_{X}$, 2) $f$ is equal to $1_{X}$ on the outside of $Y$ and 3) $f$ has no fixed points in $\stackrel{\circ}{Y}$.

We fix the following notations.

Notation 5. $B$ is the leaf space, and $B_{0}, B_{1}$ and $B_{2}$ are subsets of $B$ as is stated above. $g$ is the genus of a generic leaf $L$, and $\alpha_{i}, \beta_{i}(1 \leq i \leq g)$ are simple closed curves on $L$ such that $\left\langle\alpha_{i}, \alpha_{j}\right\rangle=0,\left\langle\beta_{i}, \beta_{j}\right\rangle=0$ and $\left\langle\alpha_{i}, \beta_{j}\right\rangle=\delta_{i j}$ for any $i, j$, where $\langle$,$\rangle denotes the algebraic intersection number of 1$-cycles in $L$. We denote by $\left[\alpha_{i}\right]$ and $\left[\beta_{i}\right]$ the homology classes of $\alpha_{i}$ and $\beta_{i}$. Then $\left[\alpha_{i}\right],\left[\beta_{i}\right](1 \leq i \leq g)$ form a canonical symplectic basis for $H_{1}(L: \mathbb{Z})$. We denote by $\left\{\alpha_{i}\right\},\left\{\beta_{i}\right\}$ the homotopy classes of $\alpha_{i}, \beta_{i}$. Then $\left\{\alpha_{i}\right\},\left\{\beta_{i}\right\}(1 \leq i \leq g)$ 
represent generators of $\pi_{1}(L)$.

\section{§2. Stability of a Foliation with All Leaves Tori}

In this section we study about perturbations of foliations with all leaves tori and singular leaves.

Proposition 6. If a foliation with all leaves tori has a rotation or dihedral leaf, then the foliation is $C^{1}$-stable.

Proof. This follows from Theorem 1.1 of Hirsch [7] since a certain linear holonomy of such a leaf has not 1 as an eigenvalue.

We consider here the case a foliation $F$ has only reflection leaves as singular leaves. Each connected component of the union $R(F)$ of reflection leaves of $F$ is diffeomorphic to $T^{2} \times[0,1] / h$, where $(x, 0)$ and $(y, 1)$ are identified by a diffeomorphism $h$ of $T^{2}$. We denote a connected component of $R(F)$ by the same letter. Let Möb be the Möbius band obtained in the product $S^{1} \times(-1,1)$ with coordinate $(\theta, u), \theta \in S^{1}=\mathbb{R} / \mathbb{Z}, u \in(-1,1)$ by identifying $(\theta, u)$ and $(\theta+1 / 2,-u)$. The foliation on $S^{1} \times(-1,1)$ with leaves of form $S^{1} \times\{p t\}$ induces a foliation $F_{1}$ on Möb. So we define a foliation $F_{2}$ on Möb $\times S^{1} \times[0,1]$ with leaves of form $L \times S^{1} \times\{p t\}, L \in F_{1}$. Let $U$ be a saturated tubular neighborhood of $R(F)$ in $M$. Then $(U, F)$ is diffeomorphic to (Möb $\left.\times S^{1} \times[0,1], F_{2}\right) / H$, where $H: \mathrm{Möb} \times S^{1} \rightarrow \mathrm{Möb} \times S^{1}$ is a foliation preserving diffeomorphism extended from $h$ and a point $p$ in $M o ̈ b \times S^{1}$ is assumed to be fixed by $H$. We take generators $\alpha$ and $\beta$ of $\pi_{1}\left(\mathrm{Möb} \times S^{1}, p\right)$ corresponding to generators of $\pi_{1}(\mathrm{Möb})$ and $\pi_{1}\left(S^{1}\right)$ respectively. Let $h_{*}: H_{1}\left(T^{2}\right) \rightarrow H_{1}\left(T^{2}\right)$ be the automorphism.

$$
\begin{aligned}
& \text { Lemma 7. } h_{*}=\left(\begin{array}{cc}
2 k+1 & l \\
2 m & 2 n+1
\end{array}\right) \text {, where } k, l, m, n \in \mathbb{Z} \text { and }(2 k+1)(2 n+1) \\
& -2 m l= \pm 1 .
\end{aligned}
$$

Proof. The holonomy along $\alpha$ is non-trivial and of order two and the holonomy along $\beta$ is trivial. So the holonomy along $h_{*}(\beta)$ is trivial, hence $h_{*}(\beta)=2 m \alpha+n^{\prime} \beta\left(m, n^{\prime} \in \mathbb{Z}\right)$. Since $h_{*}$ belongs to $G L(2, \mathbb{Z})$, diagonal components are odd numbers.

We consider the special case $k=-1$ and $n=-1$.

Theorem 8. Let $F$ and $U$ be as above. Suppose $h_{*}=\left(\begin{array}{cc}-1 & 0 \\ 0 & -1\end{array}\right)$. Then 
every sufficiently small $C^{1}$-perturbation of $F$ has a compact leaf in $U$. Hence $F$ is $C^{1}$-stable.

Proof. We may assume that $U=\mathrm{Möb} \times S^{1} \times[0,1] / H$ with coordinate $(\theta, u, \varphi, t),(\theta, u) \in \mathrm{Möb}, \varphi \in S^{1}=\mathbb{R} / \mathbb{Z}$ and $t \in[0,1]$, and $p=(0,0,0) \in \mathrm{Möb} \times S^{1}$ and a segment $E=\{(0, u, 0) ;-1<u<1\} \subset$ Möb $\times S^{1}$ are left invariant by $H$. The set $E \times[0,1] / H$ can be considered to be the set $E \times S^{1}$, if necessary, by taking an appropriate double covering $\widetilde{U}$ of $U$. For, let $F^{\prime}$ be a small $C^{1}$ perturbation of $F$. Then the foliation $\widetilde{F}^{\prime}$ induced on $\widetilde{U}$ is also a small $\mathbb{C}^{1}$ perturbation of the foliation $\widetilde{F}$ induced on $\widetilde{U}$. If $\widetilde{F}^{\prime}$ has a compact leaf in $\widetilde{U}$, then $F^{\prime}$ has a compact leaf in $U$.

Let $\alpha$ and $\beta$ be loops in $L_{p \times\{0\}}$ with base point $p \times\{0\}=(0,0,0,0)$, representing generators of $\pi_{1}\left(L_{p \times\{0\}}\right) \cong \mathbb{Z} \oplus \mathbb{Z}$ such that the holonomy along $\alpha$ (resp. $\beta$ ) is non-trivial (resp. trivial). Let $\alpha(t)$ and $\beta(t)$ be translations of $\alpha$ and $\beta$ along the curve $p \times\{t\}, t \in[0,1]$. Then we can define perturbed holonomy maps $H\left(F^{\prime}, \alpha(i)\right), H\left(F^{\prime}, \beta(t)\right): E_{\delta} \times\{t\}=\{(0, u, 0) ;-\delta<u<\delta\} \times\{t\} \rightarrow E$ $\times S^{1}$ for each $t$ and some $\delta>0$, which are imbeddings (cf. Hirsch [7], LangevinRosenberg [9] and Fukui [4]). Note that 1) $H\left(F^{\prime}, \alpha\left(t_{0}\right)\right)$ and $H\left(F^{\prime}, \beta\left(\hat{t}_{0}\right)\right)$ are extended to maps $H\left(F^{\prime}, \alpha_{t_{0}}\right)$ and $H\left(F^{\prime}, \beta_{t_{0}}\right): E_{\delta} \times\left(t_{0}-r, t_{0}+r\right) \rightarrow E \times S^{1}$ for some small $r$, which are local diffeomorphisms, 2) the extended maps $H\left(F^{\prime}\right.$, $\left.\alpha_{t_{0}}\right)$ and $H\left(F^{\prime}, \alpha_{t_{1}}\right) . H\left(F^{\prime}, \beta_{t_{0}}\right)$ and $H\left(F^{\prime}, \beta_{t_{1}}\right)$ coincide on the intersections of their domains respectively if $t_{0}$ and $t_{1}$ are close, 3) $H\left(F^{\prime}, \alpha(t)\right)$ and $H\left(F^{\prime}, \beta(t)\right)$ are $C^{1}$-close to the map $R(u, t)=(-u, t)$ and $\mathrm{id}(u, t)=(u, i)$ respectively because $F$ and $F^{\prime}$ are $C^{1}$-close and 4) $H\left(F^{\prime}, \alpha(1)\right)=H\left(F^{\prime},-\alpha(0)\right)$ and $H\left(F^{\prime}, \beta(1)\right)$ $=H\left(F^{\prime},-\beta(0)\right)$ may not coincide with $H\left(F^{\prime}, \alpha(0)\right)$ and $H\left(F^{\prime}, \beta(0)\right)$ respectively.

We put $\widetilde{S}^{1}=\mathbb{R} / 2 \mathbb{Z}$ and let $\pi: \widetilde{S}^{1} \rightarrow S^{1}$ be the double covering map defined by $\pi(\tilde{t})=\tilde{t}(\bmod 1), \tilde{t} \in \widetilde{S}^{1}$. Then there exist the maps $H_{\alpha}\left(F^{\prime}\right)$ and $H_{\beta}\left(F^{\prime}\right)$ : $E_{\delta} \times \widetilde{S}^{1} \rightarrow E \times \widetilde{S}^{1}$ extended from $H\left(F^{\prime}, \alpha(t)\right)$ and $H\left(F^{\prime}, \beta(t)\right)$ (cf. [4], [9]) respectively, such that the following diagram commutes;

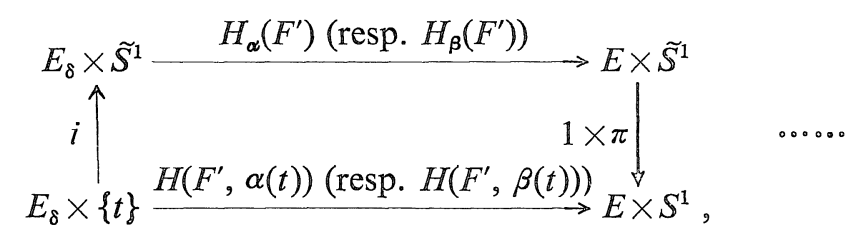

where $i((0, u, 0, t))=(0, u, 0, t)$ and $(1 \times \pi)(0, u, 0, \tilde{t}))=(0, u, 0, \pi(\tilde{t}))$. We put $H_{\alpha}\left(F^{\prime}\right)(u, \tilde{t})=\left(f_{1}(u, \tilde{t}), f_{2}(u, \tilde{t})\right)$ using the coordinate $(u, \tilde{t})$ of $E_{\delta} \times{\widetilde{S^{1}}}^{1}$. Then there exists a unique $u(\tilde{t})$ for each $\tilde{t} \in \widetilde{S}^{1}$ such that $u(\tilde{t})=f_{1}(u(\tilde{t}), \tilde{t})$. The set $\tilde{\iota}=\left\{(u(\tilde{t}), \tilde{t}) ; \tilde{t} \in \tilde{S}^{1}\right\}$ is a loop in $E \times \tilde{S}^{1}$, so $(1 \times \pi)(\tilde{l})$ is a loop in $E \times S^{1}$, which 
rotates twice around $S^{1}$. Therefore there exists a $t_{0}\left(t_{0} \in S^{1}\right)$ with $u\left(t_{0}\right)=$ $u\left(t_{0}+1\right)$. We may assume $t_{0}=0$. So the set $\left\{(u(t), t) ; t \in S^{1}\right\}$ in $E \times S^{1}$ is a loop $\ell$, which may have a corner at $(u(0), 0)$. By the same argument as in the proof of Theorem 4 of Fukui [5], there exists a point $q=\left(u\left(t_{1}\right), t_{1}\right) \in \downarrow$ such that $H\left(F^{\prime}, \alpha\left(t_{1}\right)\right)(q)=q$, that is, $q$ is a fixed point of $H_{\alpha}\left(F^{\prime}\right)$.

We consider the behavior of $H_{\beta}^{n}\left(F^{\prime}\right)(q)(n \in \mathbb{Z})$ for a fixed point $q$ of $H_{\alpha}\left(F^{\prime}\right)$.

Lemma 9. $H_{\beta}\left(F^{\prime}\right)(q)$ is a fixed point of $H_{\alpha}\left(F^{\prime}\right)$ and belongs to $\tilde{\iota}$.

Proof. $\quad H_{\alpha}\left(F^{\prime}\right) \circ H_{\beta}\left(F^{\prime}\right)=H\left(F^{\prime}, \alpha \cdot \beta\right)=H\left(F^{\prime}, \beta \cdot \alpha\right)=H_{\beta}\left(F^{\prime}\right) \circ H_{\alpha}\left(F^{\prime}\right)$. Thus $H_{\alpha}\left(F^{\prime}\right)\left(H_{\beta}\left(F^{\prime}\right)(q)\right)=H_{\beta}\left(F^{\prime}\right)\left(H_{\alpha}\left(F^{\prime}\right)(q)\right)=H_{\beta}\left(F^{\prime}\right)(q)$. Hence $H_{\beta}\left(F^{\prime}\right)(q)$ is a fixed point of $H_{\alpha}\left(F^{\prime}\right)$. Since we have a unique $u(\tilde{t})$ with $u(\tilde{t})=f_{1}(u(\tilde{t}), \tilde{t})$ for each $\tilde{t} \in{\widetilde{S^{1}}}^{1}, H_{\beta}\left(F^{\prime}\right)(q)$ lies in $\tilde{\iota}$.

For the simplicity, we put $\varphi^{\prime}=H_{\beta}\left(F^{\prime}\right)$. We denote by $\operatorname{Fix}\left(H_{a}\right)$ the fixed point set of $H_{\alpha}\left(F^{\prime}\right)$. Note that $\varphi^{\prime}\left(\operatorname{Fix}\left(H_{\alpha}\right)\right)=\operatorname{Fix}\left(H_{\alpha}\right)$ and $\varphi^{\prime}\left(\operatorname{Fix}\left(H_{\alpha}\right)\right) \subset \tilde{l}$ by Lemma 9.

Let $\tilde{\boldsymbol{\pi}}: E \times \widetilde{\boldsymbol{S}}^{1} \rightarrow \tilde{\iota}$ be the map defined by $\tilde{\boldsymbol{\pi}}(u, \tilde{t})=(u(\tilde{t}), \tilde{t})$ and $\varphi=\tilde{\boldsymbol{\pi}} \circ \varphi^{\prime} \mid \tilde{\iota}$ : $\tilde{\ell} \rightarrow \tilde{l}$. Then we easily see that $\varphi$ is a diffeomorphism of $\tilde{\ell}$ and $\varphi$ and $\varphi^{\prime}$ coincide on $\operatorname{Fix}\left(H_{\alpha}\right)$.

Proposition 10. There exists a point $p$ in $\tilde{l}$ such that $p$ is a fixed point of $H_{\alpha}\left(F^{\prime}\right)$ and a periodic point of $H_{\beta}\left(F^{\prime}\right)$.

We prove Proposition 10 as follows. We suppose that $\varphi$ has no periodic points on $\operatorname{Fix}\left(H_{a}\right)$. We introduce on $\tilde{\iota}$ a fixed orientation. If $a$ and $b$ are different points of $\tilde{l}$, the $\overparen{a b}$ denotes the oriented simple arc connecting $a$ with $b$, and the formula $a<c<b$ means that the point $c$ lies on the arc $\overparen{a b}$. Since $\varphi$ is $C^{1}$-close to the identity, $\varphi$ preserves the orientation.

Then we can prove Lemma 11 and use it to prove Lemma 12 similarly as in the proofs of Lemmas 1 and 2 of Siegel [14].

Lemma 1 耳. Let $q$ be a point in Fix $\left(H_{\alpha}\right)$. For a non-zero integer $m$ be given, then there exists an integer $h$ such that $q<\varphi^{h}(q)<\varphi^{m}(q)$.

We suppose that a point $p \in \operatorname{Fix}\left(H_{\alpha}\right)$ is not ergodic, that is, the orbit set $O(p)=\left\{\varphi^{n}(p) ; n \in \mathbb{Z}\right\}$ is not dense in $\tilde{\ell}$.

$\tilde{l}-\overline{O(p)}$ is an open and non-empty set. Choose in $\tilde{l}-\overline{O(p)}$ an open arc $\overparen{a b}$ whose end points belong to $\overline{O(p)}$. The end points $a_{n}, b_{n}$ of all images arcs ${\widetilde{a_{n} b_{n}}}_{=} \varphi^{n}(\widehat{a b})(n \in \mathbb{Z})$ lie in $\overline{O(p)}$ and the inner points of these arcs lie in $\tilde{\iota}-\overline{O(p)}$, 
hence $\overparen{a_{n} b_{n}}(n \in \mathbb{Z})$ are disjoint.

Lemma 12. Let $a_{n}, b_{n}(n \in \mathbb{Z})$ be as above. For an arbitrarily large natural number $N$, there exists an integer $m>N$ such that either the m-arcs $\overbrace{a_{-k} b_{m-k}}$ or $\overbrace{a_{m-k} b_{-k}}(k=1,2, \cdots, m)$ are disjoint.

By the similar argument as in Siegel [14], Lemma 12 leads us to a contradiction. Hence $O(p)$ is dense in $\tilde{\ell}$. This implies Fix $\left(H_{\alpha}\right)=\tilde{l}$. We put $\varphi^{\prime}(u, \tilde{t})=\left(g_{1}(u, \tilde{t}), g_{2}(u, \tilde{t})\right)$ for $(u, \tilde{t}) \in E_{\delta} \times \widetilde{S}^{1}$. From $(*), H\left(F^{\prime}, \beta(t)\right)(u, t)=$ $\left(g_{1}(u, t), g_{2}(u, t)\right)$. Since we suppose that $\varphi^{\prime}$ has no periodic points on $\operatorname{Fix}\left(H_{\alpha}\right)$, the point $(u(0), 0)$ is not a fixed point of $\varphi^{\prime}$ and if $g_{2}(u(0), 0)>0$, then $g_{2}(u(1), 1)$ $>1$. On the other hand, $g_{2}(u(1), 1)<1$ because $H\left(F^{\prime}, \beta(1)\right)=H\left(F^{\prime},-\beta(0)\right)$. This is a contradiction. Hence we have Proposition 10.

The following proposition is proved by the standard argument (cf. Langevin-Rosenberg [8]).

Proposition 13. If $p$ in $\tilde{l}$ is a fixed point of $H_{\alpha}\left(F^{\prime}\right)$ and a periodic point of $H_{\beta}\left(F^{\prime}\right)$, then $L_{p}^{\prime}$ is compact, where $L_{p}^{\prime}$ is a leaf of $F^{\prime}$ through $p$.

We complete the proof of Theorem 8 by Propositions 10 and 13 .

Remark 14. Theorem 8 holds for $C^{2}$-foliations.

Theorem 15. Let $F$ and $U$ be as above. Suppose $h_{*}=\left(\begin{array}{cc} \pm 1 & l \\ 0 & 1\end{array}\right)$ or $\left(\begin{array}{cc}1 & 0 \\ 2 m & \pm 1\end{array}\right)$ $(\ell, m \in \mathbb{Z})$. Then there is a foliation $F^{\prime}$ such that $F^{\prime}$ is $C^{r}$-close to $F$ and $F^{\prime}$ has no compact leaves in $U$.

Proof. We prove the case $h_{*}=\left(\begin{array}{cc} \pm 1 & l \\ 0 & 1\end{array}\right)$. It is proved similarly for the case $h_{*}=\left(\begin{array}{cc}1 & 0 \\ 2 m & \pm 1\end{array}\right)$. We consider the product $\mathrm{Möb} \times S^{1} \times \mathbb{R}$ with coordinate $(\theta, u, \varphi, t),(\theta, u) \in \mathrm{Möb}, \varphi \in S^{1}$ and $t \in \mathbb{R}$, and define a foliation $G$ on Möb $\times S^{1} \times \mathbb{R}$ to be the set of leaves whose tangent spaces are spanned by $\frac{\partial}{\partial \theta}$ and $\frac{\partial}{\partial \varphi}$. Let $\hat{H}: \operatorname{Möb} \times S^{1} \times \mathbb{R} \rightarrow \operatorname{Möb} \times S^{1} \times \mathbb{R}$ be a diffeomorphism defined by $\hat{H}(\theta, u, \varphi, t)=( \pm \theta+l \circ \varphi, \varepsilon u, \varphi, t+1), \varepsilon= \pm 1$. Then $(U, F)$ is diffeomorphic to $\left(\mathrm{Möb} \times S^{1} \times \mathbb{R}, G\right) / \hat{H}$. Now we define a new foliation $G^{\prime}$ on $\mathrm{Möb} \times S^{1} \times \mathbb{R}$ to be the set of leaves whose tangent spaces are spanned by $\frac{\partial}{\partial \theta}$ and $\frac{\partial}{\partial \varphi}+\lambda \frac{\partial}{\partial t}$, where $\lambda$ is a small irrational number. Then $\hat{H}$ preserves $G^{\prime}$ because $\hat{H}_{*}\left(\frac{\partial}{\partial \theta}\right)= \pm \frac{\partial}{\partial \theta}$ 
and $\hat{H}_{*}\left(\frac{\partial}{\partial \varphi}+\lambda \frac{\partial}{\partial t}\right)=\frac{\partial}{\partial \varphi}+l \frac{\partial}{\partial \theta}+\lambda \frac{\partial}{\partial t}$. Hence we can define a foliation $F_{1}=$ $G^{\prime} / \hat{H}$ on $\mathrm{Möb} \times S^{1} \times \mathbb{R} / \hat{H}$. We can easily extend $F_{1}$ to $F^{\prime}$ on $M$ such that $F$ and $F^{\prime}$ are $C^{r}$-close $(r \geq 0)$. It is easy to see that $F^{\prime}$ has no compact leaves in $U$.

\section{§3. Instability of Folliations withoun Simgular Leaves}

In this section we consider the case $F$ has no singular leaves, that is, $\pi$ : $M \rightarrow B$ is a fibre bundle. To begin with we show the following which is true for arbitrary closed manifolds $B$.

Proposition 16. If the bundle $\pi: M \rightarrow B$ is trivial and $g \geq 2$, then $F$ is $C^{r}$ unstable $(r \geq 0)$.

Proof. Take diffeomorphisms $f_{1}$ and $f_{2}$ of $B$ such that $f_{1}$ and $f_{2}$ are sufficiently $C^{r}$-close to $1_{B}$ and the periodic point sets of $f_{1}$ and $f_{2}$ are disjoint. Then we define a homomorphism $\Phi: \pi_{1}(L) \rightarrow \operatorname{Diff}(B)$ by $\Phi\left(\left\{\alpha_{i}\right\}\right)=f_{i}(i=1,2)$, $\Phi\left(\left\{\alpha_{i}\right\}\right)=1_{B}(i=3,4, \cdots, g)$ and $\Phi\left(\left\{\beta_{i}\right\}\right)=1_{B}(i=1,2, \cdots, g)$. This defines a foliation $F^{\prime}$ of $M=L \times B$ whose leaves are transverse to the fibres of another fibre bundle $\pi^{\prime}: M \rightarrow L$ with fibre $B$. From the properties of $f_{i}$, we see that $F^{\prime}$ is sufficiently $C^{r}$-close to $F$ and has no compact leaves.

Theorem 17. (1) If $B$ is homeomorphic to the 2-sphere $S^{2}$ and $g \geq 2$, then $F$ is $C^{r}$-unstable $(r \geq 0)$.

(2) If $B$ is homeomorphic to the projective plane $P^{2}$ and $g \geq 4$, then $F$ is $C^{\gamma}$. unstable $(r \geq 0)$.

(3) If $B$ is neither homeomorphic to $S^{2}$ nor $P^{2}$ and $g \geq 6$, then $F$ is $C^{r}$-unstable $(r \geq 0)$.

Proof of (1). In this case, it is an immediate consequence of Proposition 16 because that any bundle over $S^{2}$ with fibre $L$ of genus $\geq 2$ is trivial.

Proof of (2). Any bundle over $P^{2}$ with fibre $L$ of genus $\geq 2$ is obtained by making the identifications in $L \times D^{2}$ as follows: $(x, y) \sim(\varphi(x),-y)$ for $x \in L, y \in D^{2}$, where $\varphi: L \rightarrow L$ is a diffeomorphism with $\varphi^{2}=1_{L}$.

Step 1. We perturb $F$ on $\pi^{-1}\left(\stackrel{\circ}{D}^{2}\right) \cong L \times \stackrel{\circ}{D^{2}}$ as follows. Let $G: D^{2} \rightarrow D^{2}$ be a diffeomorphism satisfying the property $P\left(D^{2}, r\right)$. Let $Q$ be an open tubular neighborhood of $\alpha_{1}$ in $L$ such that its closure $\bar{Q}$ is homeomorphic to $\alpha_{1}$ $\times[0,1]$ with coordinate $(s, t), s \in \alpha_{1}, 0 \leq t \leq 1$. We start with the foliation of $(L-Q) \times \stackrel{\circ}{D}^{2}$ having leaves of form $(L-Q) \times\{y\}, y \in \stackrel{\circ}{D}^{2}$ and make the identi- 
fications $(s, 0, y) \sim(s, 1, G(y))$ to obtain a foliation of $L \times \stackrel{\circ}{D}^{2}$ having no compact leaves. Replacing $F$ on $\pi^{-1}\left(\stackrel{\circ}{D}^{2}\right)$ by this foliation yields a new foliation $F^{\prime}$ which has no compact leaves on $\pi^{-1}\left(\stackrel{\circ}{D}^{2}\right)$.

Step 2. Take a point $p$ of $\partial D^{2} / \sim$ and a conic tubular neighborhood $A$ of $\partial D^{2} / \sim-p$ in $D^{2} / \sim$ as in Fig. 2 .

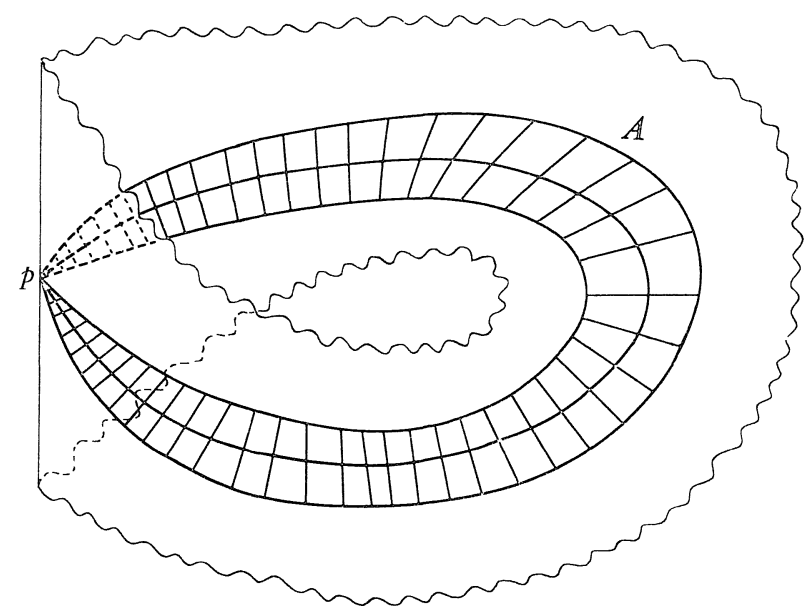

Figure 2

We want to perturb $F^{\prime}$ on $\pi^{-1}(A) \cong L \times A$ to obtain a foliation $F^{\prime \prime}$ such that 1) $F^{\prime \prime}$ is sufficiently $C^{r}$-close to $\left.F, 2\right) F^{\prime \prime}$ has no compact leaves on $\pi^{-1}\left(P^{2}-p\right)$ and 3) $L_{p}=\pi^{-1}(p)$ is the only compact leaf of $F^{\prime \prime}$. Since $g \geq 4$, there exists a simple closed curve $\delta$ on $L$ in $\pi^{-1}\left(\partial D^{2} / \sim\right)$ such that $[\delta] \neq 0$ in $H_{1}(L ; \mathbb{Z})$ and $\left\langle\delta, \alpha_{1}\right\rangle=\left\langle\delta, \varphi\left(\alpha_{1}\right)\right\rangle=0$ (see Plante [10]). In fact $\left\langle\alpha_{2}, \varphi\left(\alpha_{1}\right)\right\rangle \beta_{2}-\left\langle\beta_{2}, \varphi\left(\alpha_{1}\right)\right\rangle \alpha_{2}$ is homologous to a multiple of a simple closed curve. Let $\gamma$ be an arbitrary closed curve on $L$. Then the holonomy of $L$ in $F^{\prime}$ along $r$ is trivial if and only if $\left\langle\gamma, \alpha_{1}\right\rangle=\left\langle\gamma, \varphi\left(\alpha_{1}\right)\right\rangle=0$. Hence the holonomy of $L$ in $F^{\prime}$ along $\delta$ is trivial. Take a tubular neighborhood $Q_{\delta}$ of $\delta$ in $L$ such that its closure $\bar{Q}_{\delta}$ is homeomorphic to $\delta \times[0,1]$ with coordinate $(s, t), s \in \delta, 0 \leq t \leq 1$. Take a diffeomorphism $H: P^{2} \rightarrow P^{2}$ satisfying the property $P(A, r) . \quad F^{\prime}$ restricted to $\bar{Q}_{\delta} \times A$ has leaves of form $\delta \times[0,1] \times\{y\}, y \in A$. Thus we start with the foliation of $\delta \times[0,1] \times A-\delta \times(1 / 3,2 / 3) \times A$ having leaves of form $\delta \times[0,1 / 3] \cup[2 / 3,1]$ $\times\{y\}, y \in A$ and make the identifications $(s, 1 / 3, y) \sim(s, 2 / 3, H(y))$ to obtain a foliation of $\bar{Q}_{\delta} \times A$. Replacing $F^{\prime}$ on $\bar{Q}_{\delta} \times A$ by this foliation, we obtain a required foliation $F^{\prime \prime}$.

Step 3. Finally we perturb $F^{\prime \prime}$ on a neighborhood of the compact leaf $L_{p}$. Since $g \geq 4$, there is a simple closed curve $\eta$ on $L_{p}$ such that $[\eta] \neq 0$ in 
$H_{1}\left(L_{p} ; \mathbb{Z}\right)$ and $(1)\left\langle\eta, \alpha_{1}\right\rangle=\langle\eta, \delta\rangle=0,(2)\left\langle\eta, \varphi\left(\alpha_{1}\right)\right\rangle=\langle\eta, \varphi(\delta)\rangle=0$. For, put $\eta=\sum_{i=3}^{g} m_{i} \alpha_{i}+\sum_{i=3}^{g} n_{i} \beta_{i}\left(m_{i}, n_{i} \in \mathbb{Z}\right)$. Then the equations (1) are satisfied. So we consider the equations (2). We can solve (2) over integers since $g \geq 4$. Let $\eta_{0}$ be a non-trivial general solution of (2). Then we can choose a simple closed curve $\eta$ such that $\eta_{0}$ is homologous to a multiple of $\eta$ because $\eta=\sum_{i=3}^{g} m_{i} \alpha_{i}+\sum_{i=3}^{g} n_{i} \beta_{i}$ is realizable by a simple closed curve on $L_{p}$ if $m_{3}, \cdots, m_{g}, n_{3}, \cdots, n_{g}$ are relatively prime. The holonomy of $L_{p}$ in $F^{\prime \prime}$ along $\eta$ is trivial. Hence the rest of the proof is done similarly as in Step 2 .

Proof of (3). We prove the case B is orientable. It is proved similarly for the non-orientable case. Note that $B_{1}$ is a closed surface in this case.

Lemma 18. There exists a foliation $F_{1}$ of $M$ such that 1) $F_{1}$ is sufficiently $C^{r}$-close to $\left.F, 2\right) F_{1}$ has no compact leaves on $\pi^{-1}\left(B-\left\{p_{1}, \cdots, p_{2 h-1}\right\}\right)$ and 3$)$ $L_{p_{i}}=\pi^{-1}\left(p_{i}\right)(i=1,2, \cdots, 2 h-1)$ are the compact leaves of $F_{1}$.

Proof. First we perturb $F$ on $\pi^{-1}\left(\stackrel{\circ}{B}_{2}\right) \cong L \times \stackrel{\circ}{B}_{2}$ as in Step 1 in the proof of (2) using $\alpha_{1}$ on $L$ and a diffeomorphism of $B$ satisfying the property $P\left(B_{2}, r\right)$. We let $F^{\prime}$ be the resulting foliation. Next take conic tubular neighborhoods $A_{1}, A_{i j}(i=2,3, \cdots, 2 h-1 ; j=1,2)$ and $A_{2 h, 1}$ of $c_{1}^{\prime}, c_{i j}$ and $c_{2 h, 1}$ such that they are disjoint and the closures of $A_{1}$ and $A_{2 j}$ have the only point $p_{1}$ in common $(j=1,2)$ and the closures of $A_{i 1}$ and $A_{i 2}(i=2, \cdots, 2 h-1)$ have the points $p_{i-1}$ and $p_{i}$ in common and the closures of $A_{2 h-1, j}$ and $A_{2 h, 1}$ have the only point $p_{2 h-1}$ in common $(j=1,2)$ (see Fig. 3).

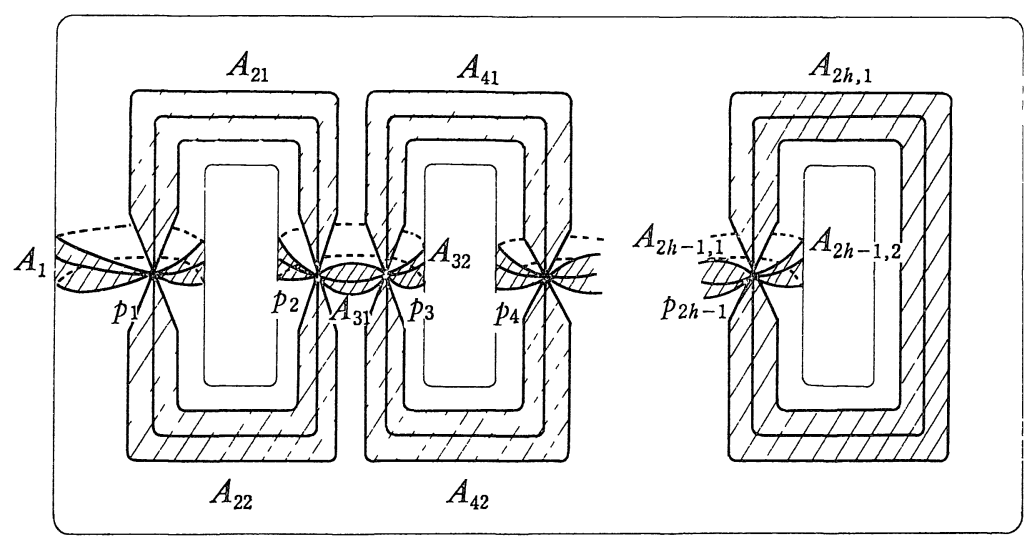

Figure 3

There exist simple closed curves $\delta_{1}, \delta_{i j}(i=2, \cdots, 2 h-1 ; j=1,2)$ and $\delta_{2 h, 1}$ 
on the compact leaves $L$ of $F^{\prime}$ in $\pi^{-1}\left(A_{1}\right), \pi^{-1}\left(A_{i j}\right)$ and $\pi^{-1}\left(A_{2 h, 1}\right)$ respectively such that $\left[\delta_{1}\right] \neq 0,\left[\delta_{i j}\right] \neq 0$ and $\left[\delta_{2 h, 1}\right] \neq 0$ in $H_{1}(L ; \mathbb{Z})$ and $\left\langle\delta_{1}, \alpha_{1}\right\rangle=\left\langle\delta_{1}, \varphi_{1}\left(\alpha_{1}\right)\right\rangle$ $=0,\left\langle\delta_{i j}, \alpha_{1}\right\rangle=\left\langle\delta_{i j}, \varphi_{i j}\left(\alpha_{1}\right)\right\rangle=0(i=2,3, \cdots, 2 h-1 ; j=1,2)$ and $\left\langle\delta_{2 h, 1}, \alpha_{1}\right\rangle$ $=\left\langle\delta_{2 h, 1}, \varphi_{2 h, 1}\left(\alpha_{1}\right)\right\rangle=0$ (see Plante [10]). $\left[\delta_{1}\right],\left[\delta_{i j}\right]$ and $\left[\delta_{2 h, 1}\right]$ can be considered to be expressed as linear combinations of $\left[\alpha_{2}\right]$ and $\left[\beta_{2}\right]$. Take diffeomorphisms $G_{1}, G_{i j}(i=2,3, \cdots, 2 h-1 ; j=1,2)$ and $G_{2 h, 1}: B \rightarrow B$ satisfying the properties $P\left(A_{1}, r\right), P\left(A_{i j}, r\right)$ and $P\left(A_{2 h, 1}, r\right)$ respectively. Then, by the similar argument to Step 2 in the proof of (2), we can perturb $F^{\prime}$ on $\pi^{-1}\left(A_{1}\right) \cong L \times A_{1}, \pi^{-1}\left(A_{i j}\right)$ $\cong L \times A_{i j}$ and $\pi^{-1}\left(A_{2 h, 1}\right) \cong L \times A_{2 h, 1}$ using $\delta_{1}, \delta_{i j}$ and $\delta_{2 h, 1}$ to obtain a required foliation $F_{1}$.

Lemma 19. There exist simple closed curves $\eta_{i}$ on $L_{p_{i}}(i=1,2, \cdots, 2 h-1)$ such that $\left[\eta_{i}\right] \neq 0$ in $H_{1}\left(L_{p_{i}} ; \mathbb{Z}\right)$ and

$$
\begin{aligned}
& (1-1)\left\langle\eta_{1}, \alpha_{1}\right\rangle=\left\langle\eta_{1}, \delta_{1}\right\rangle=\left\langle\eta_{1}, \delta_{2 j}\right\rangle=0(j=1,2) \\
& (1-2)\left\langle\eta_{1}, \varphi_{1}\left(\delta_{1}\right)\right\rangle=\left\langle\eta_{1}, \varphi_{2 j}\left(\delta_{2 j}\right)\right\rangle=0(j=1,2) \\
& (1-3)\left\langle\eta_{1}, \varphi_{1}\left(\alpha_{1}\right)\right\rangle=\left\langle\eta_{1}, \varphi_{22} \circ \varphi_{1}\left(\alpha_{1}\right)\right\rangle=\left\langle\eta_{1}, \varphi_{1}^{-1} \circ \varphi_{22} \circ \varphi_{1}\left(\alpha_{1}\right)\right\rangle=0 \\
& (i-1)\left\langle\eta_{i}, \alpha_{1}\right\rangle=\left\langle\eta_{i}, \delta_{i j}\right\rangle=\left\langle\eta_{i}, \delta_{i+1, j}\right\rangle=0(j=1,2) \\
& (i-2)\left\langle\eta_{i}, \varphi_{i j}\left(\delta_{i j}\right)\right\rangle=\left\langle\eta_{i}, \varphi_{i+1, j}\left(\delta_{i+1, j}\right)\right\rangle=0(j=1,2) \\
& (i-3)\left\langle\eta_{i}, \varphi_{i 1}\left(\alpha_{1}\right)\right\rangle=\left\langle\eta_{i}, \varphi_{i+1,1} \circ \varphi_{i 1}\left(\alpha_{1}\right)\right\rangle=\left\langle\eta_{i}, \varphi_{i 2}^{-1} \circ \varphi_{i+1,1} \circ \varphi_{i 1}\left(\alpha_{1}\right)\right\rangle=0 \\
& \quad(i=2, \cdots, 2 h-2), \\
& (2 h-1-1)\left\langle\eta_{2 h-1}, \alpha_{1}\right\rangle=\left\langle\eta_{2 h-1}, \delta_{2 h-1, j}\right\rangle=\left\langle\eta_{2 h-1}, \delta_{2 h, 1}\right\rangle=0(j=1,2) \\
& (2 h-1-2)\left\langle\eta_{2 h-1}, \varphi_{2 h-1, j}\left(\delta_{2 h-1, j}\right)\right\rangle=\left\langle\eta_{2 h-1}, \varphi_{2 h, 1}\left(\delta_{2 h, 1}\right)\right\rangle=0(j=1,2) \\
& (2 h-1-3)\left\langle\eta_{2 h-1}, \varphi_{2 h-1,1}\left(\alpha_{1}\right)\right\rangle=\left\langle\eta_{2 h-1}, \varphi_{2 h, 1} \circ \varphi_{2 h-1,1}\left(\alpha_{1}\right)\right\rangle \\
& =\left\langle\eta_{2 h-1}, \varphi_{2 h-1,1}^{-1} \circ \varphi_{2 h} \circ \varphi_{2 h-1,1}\left(\alpha_{1}\right)\right\rangle=0
\end{aligned}
$$

Proof. We prove for each $i$. Put $\eta=\sum_{i=3}^{g} m_{i} \alpha_{i}+\sum_{i=3}^{g} n_{i} \beta_{i}\left(m_{i}, n_{i} \in \mathbb{Z}\right)$. Then the equations $(i-1)$ are satisfied. So we consider the system equations $(i-2)$ and $(i-3)$. If $g \geq 6$, we can solve the equations $(i-2)$ and $(i-3)$ over integers. Let $\eta_{0}$ be a non-trivial general solution of $(i-2)$ and $(i-3)$. Then we can choose a simple closed curve $\eta$ such that $\eta_{0}$ is homologous to a multiple of $\eta$ because $\eta=\sum_{i=3}^{g} m_{i} \alpha_{i}+\sum_{i=3}^{g} n_{i} \beta_{i}\left(m_{i}, n_{i} \in \mathbb{Z}\right)$ is realizable by a simple closed curve on $L_{p_{i}}$ if $m_{3}, \cdots, m_{g}, n_{3}, \cdots, n_{g}$ are relatively prime.

Proof of Theorem 17 (3) continued. By Lemma 18, it is sufficient to perturb the compact leaves $L_{p_{i}}(i=1,2, \cdots, 2 h-1)$. Take small neighborhoods $C_{i}$ of $p_{i}$ such that they are disjoint. From Lemma 19, there exist simple closed curves $\eta_{i}$ on $L_{p_{i}}$ satisfying the conditions (i-1), (i-2) and $(i-3)$. Thus 
by the similar argument to Step 3 in the proof of (2), we can perturb $F_{1}$ on $\pi^{-1}\left(C_{i}\right)$ using $\eta_{i}$ to obtain a foliation which has no compact leaves. This completes the proof.

\section{§4. Instability of Foliations with Singular Leaves}

In this section we consider the case $F$ has singular leaves.

Proposition 20. Let $F$ be a foliation of $M$ such that $B_{0}$ has $m+n$ boundaries. If $g \geq 2$, then there exists a foliation $F_{1}$ of $M$ such that $F_{1}$ is sufficiently $C^{r}$-close to $F$ and $F_{1}$ has no compact leaves on $\pi^{-1}\left(\stackrel{\circ}{B}_{0}-\left\{p_{1}, \cdots, p_{s}\right\}\right)$, where $s=2 h+m+n-1$ if $B$ is an orientable surface of genus $h$ and $s=2 h_{1}+m+n$ if $B$ is homeomorphic to $\sum_{h_{1}} \# P^{2}-\bigcup_{i=1}^{m+n} D_{i}^{2}$ or $\sum_{h_{1}} \# K^{2}-\bigcup_{i=1}^{m+n} \stackrel{\circ}{D}_{i}^{2}$ for an orientable surface of genus $h_{1}, \Sigma_{h_{1}}$.

Proof. It is similarly proved as in the proof of Lemma 18. Moreover we can perturb $F$ on $\pi^{-1}\left(\stackrel{\circ}{B}_{2}\right)$ using $\alpha_{1}, \pi^{-1}\left(\stackrel{\circ}{c}_{1}\right)$ using $\delta_{1}, \pi^{-1}\left(\stackrel{\circ}{c}_{i j}\right)$ using $\delta_{i j}(i=2$, $\cdots, 2 h-2 ; j=1,2), \pi^{-1}\left(\stackrel{\circ}{c}_{2 h-1, j}\right)$ using $\delta_{2 h-1, j}(j=1, \cdots, m+n+1)$ and $\pi^{-1}\left(\stackrel{\circ}{d}_{j}\right)$

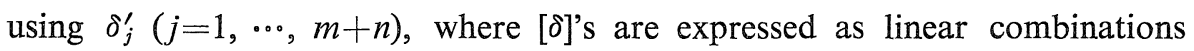
of $\left[\alpha_{2}\right]$ and $\left[\beta_{2}\right]$.

A) Case of foliations with only rotation leaves as singular leaf.

Note that $F$ is a foliation which satisfies $n=0$ in $\S 1$ and $B$ is a $V$-manifold without boundary.

Theorem 21. Let $F$ be a foliation of $M$ such that $F$ has $m$ rotation leaves $L_{1}, \cdots, L_{m}$ with holonomy order $k_{1}, \cdots, k_{m}$ respectively. If $g \geq 3 \max \left(k_{i} ; 1 \leq i \leq m\right)$ +1 , then $F$ is $C^{r}$-unstable $(r \geq 0)$.

Proof. First we perturb $F_{1}$ in Proposition 20 on each $U\left(L_{j}\right) \cong \pi^{-1}\left(V_{j}\right)$. Let $\bar{\alpha}_{1}=p_{j}\left(\alpha_{1}\right)$ and $\bar{\delta}_{j}^{\prime}=p_{j}\left(\delta_{j}^{\prime}\right)(j=1, \cdots, m)$, where $p_{j}: L \rightarrow L_{j}$ is the covering map. Let $\tau_{j}(j=1,2, \cdots, m)$ be simple closed curves on $L_{j}$ representing generators of the holonomy groups $\mathbb{Z}_{k_{j}}$ of $L_{j}$. If $g\left(L_{j}\right) \geq 4$, then there exist simple closed curves $\eta_{j}$ on $L_{j}$ such that $\left[\eta_{j}\right] \neq 0$ in $H_{1}\left(L_{j} ; \mathbb{Z}\right)$ and $\left\langle\eta_{j}, \bar{\alpha}_{1}\right\rangle=\left\langle\eta_{j}, \bar{\delta}_{j}^{\prime}\right\rangle$ $=\left\langle\eta_{j}, \tau_{j}\right\rangle=0$ respectively. Note that $p_{j} \circ \psi_{j}\left(\alpha_{1}\right)=p_{j}\left(\alpha_{1}\right)$. Take tubular neighborhoods $S_{j}$ of $\eta_{j}$ in $L_{j}$ which are homeomorphic to $\eta_{j} \times(0,1)$ respectively. By Proposition 3 (a) of Vogt [17], the normal disk bundles $U\left(L_{j}\right)$ are trivial. Hence the bundles over $S_{j}(j=1,2, \cdots, m)$ are the products $S_{j} \times D^{2}$ respectively. Thus by the similar argument to Step 2 in the proof of Theorem 17 (2), we 
perturb $F_{1}$ on $U\left(L_{j}\right)$ to obtain a foliation $F_{2}$ which has no compact leaves on $\pi^{-1}\left(B-\left\{p_{1}, \cdots, p_{s}\right\}\right)$. We easily see that $g\left(L_{j}\right) \geq 4$ if and only if $g \geq 3 k_{j}+1$ $(j=1, \cdots, m)$. Finally we perturb $L_{p_{i}}=\pi^{-1}\left(p_{i}\right)(i=1, \cdots, s)$ similarly as in Step 3 of the proof of Theorem 17 (2). For this purpose, it is sufficient to take simple closed curves $\gamma_{i}$ on $L_{p_{i}}(i=1, \cdots, s)$ such that the holonomy along each $\gamma_{i}$ is trivial. This is possible because $3 \max \left(k_{i} ; 1 \leq i \leq m\right)+1 \geq 6$ (see Lemma 19). This completes the proof.

B) Case of foliations with only reflection leaves as singullan leat。

Note that $F$ is a foliation which satisfies $m=0, n=n^{\prime \prime}$ in $\S 1$ and $B$ is a smooth manifold with $n$ boundary components.

Theorem 22. If $g \geq 7$, then $F$ is $C^{r}$-unstable $(r \geq 0)$.

Proof. We take conic tubular neighborhoods $A_{j}$ of $\partial_{j} B-q_{j}(j=1, \cdots, n)$ in $B$ and want to perturb $F_{1}$ in Proposition 20 on each $\pi^{-1}\left(A_{j}\right)$ which is homeomorphic to $L \times \stackrel{\circ}{D^{2}}$, where for $g \in \mathbb{D}, g \neq 1, g: \stackrel{\circ}{D^{2}} \rightarrow \stackrel{\circ}{D^{2}}$ is defined by $g(x, y)=(x$, $-y$ ) for $(x, y) \in \stackrel{\circ}{D}^{2}$. Let $p: L \rightarrow L_{0}(=L \times\{0\} / \mathbb{D}) \subset \pi^{-1}\left(\partial_{j} B-q_{j}\right)$ be the covering map and $\bar{\alpha}_{1}=p\left(\alpha_{1}\right)$. We put $\bar{\alpha}_{1}=\bar{\alpha}$ if $p\left(\alpha_{1}\right)$ is homologous to a twice of a simple closed curve $\bar{\alpha}$. We take a simple closed curve $\delta_{j}^{\prime \prime}$ on $L_{0}$ such that $\left[\delta_{j}^{\prime \prime}\right] \neq 0$ in $H_{1}\left(L_{0} ; \mathbb{Z}\right)$ and $\left\langle\delta_{j}^{\prime \prime}, \bar{\alpha}_{1}\right\rangle=0$. We can assume that $\left[\delta_{j}^{\prime \prime}\right]$ is expressed as a linear combination of $\left[\bar{\alpha}_{2}\right]$ and $\left[\bar{\beta}_{2}\right]$, where $\left[\bar{\alpha}_{i}\right]$ and $\left[\bar{\beta}_{i}\right]$ form a symplectic basis for $H_{1}\left(L_{0} ; \mathbb{Z}\right)$. Then taking a diffeomorphism $G_{j}: \stackrel{\circ}{D^{2}} \rightarrow \stackrel{\circ}{D^{2}}$ satisfying the property $P\left(D^{2}, r\right)$ and $G_{j}(x, y)=\left(G_{j}^{1}(x, y), y\right), G_{j}^{1}(x,-y)=G_{j}^{1}(x, y)$, we can perturb $F_{1}$ on $\pi^{-1}\left(A_{j}\right)$ using $\delta_{j}^{\prime \prime}$ to obtain a foliation $F_{2}$ which has no compact leaves on $\pi^{-1}\left(B-\left\{p_{1}, \cdots, p_{s}, q_{1}, \cdots, q_{n}\right\}\right)$. We can perturb the compact leaves $L_{p_{i}}=\pi^{-1}\left(p_{i}\right)(i=1, \cdots, s)$ in the usual way. Finally we want to perturb the compact leaves $L_{q_{j}}=\pi^{-1}\left(q_{j}\right)(j=1, \cdots, n)$. It is sufficient to take simple closed curves $\eta_{j}$ on $L_{q_{j}}$ such that $\left[\eta_{j}\right] \neq 0$ in $H_{1}\left(L_{q}, ; \mathbb{Z}\right)$ and $\left\langle\eta_{j}, \bar{\alpha}_{1}\right\rangle=\left\langle\eta_{j}, p\left(\delta_{j}^{\prime}\right)\right\rangle=$ $\left\langle\eta_{j}, \delta_{j}^{\prime \prime}\right\rangle=\left\langle\eta_{j}, \psi_{j}\left(\bar{\alpha}_{1}\right)\right\rangle=\left\langle\eta_{j}, \psi_{j}\left(\delta_{j}^{\prime \prime}\right)\right\rangle=0$. This is possible because of $g\left(L_{0}\right) \geq 4$. $g\left(L_{0}\right) \geq 4$ if and only if $g \geq 7$. This completes the proof.

\section{C) Case of coliations with dihedral leaves.}

We consider the case $F$ has no rotation leaves and some points of each boundary $\partial_{j} B$ of $B$ correspond to dihedral leaves $L_{j, k}$ with holonomy groups $\mathbb{D}_{l, k}$.

Theorem 23. If $g \geq 8 \max \left(l_{j, k} ; 1 \leq j \leq n ; 1 \leq k \leq m_{j}\right)+1$, then $F$ is $C^{r}$ unstable $(r \geq 0)$. 
Proof. We can perturb $F_{1}$ in Proposition 20 on each $\pi^{-1}\left(\partial_{j} B\right)-\bigcup_{m_{j}}^{m_{j}} L_{j} L_{j, k}$ using a simple closed curve $\delta_{j, k}^{\prime \prime}$ on a reflection leaf in $\pi^{-1}\left(\partial_{j} B\right)-\bigcup_{k=1} L_{j, k}$ as in the proof of Theorem 22, where $\left[\delta_{j, k}^{\prime \prime}\right]$ can be considered to be expressed as linear combinations of $\left[\bar{\alpha}_{2}\right]$ and $\left[\bar{\beta}_{2}\right]$. We let $F_{2}$ be the resulting foliation. Next we want to perturb $F_{2}$ on saturated tubular neighborhoods $U\left(L_{j, k}\right)$ of $L_{j, k}$. Let $p_{j, k}: L \rightarrow L_{j, k}$ be the covering map and $\lambda$ and $\mu$ simple closed curves on $L_{j, k}$ which represent generators of the holonomy group of $L_{j, k}$. We need the following lemma.

Lemma 24. There exist simple closed curves $\eta_{j, k}$ on $L_{j, k}$ such that 1) $\left[\eta_{j, k}\right]$ $\neq 0$ in $\left.H_{1}\left(L_{j, k} ; \mathbb{Z}\right), 2\right)\left\langle\eta_{j, k}, p_{j, k}\left(\alpha_{1}\right)\right\rangle=\left\langle\eta_{j, k}, p_{j, k}\left(\bar{\alpha}_{2}\right)\right\rangle=\left\langle\eta_{j, k}, p_{j, k}\left(\bar{\beta}_{2}\right)\right\rangle=\left\langle\eta_{j, k}, \lambda\right\rangle$ $=\left\langle\eta_{j, k}, \mu\right\rangle=0$ and 3) $U\left(L_{j, k}\right)$ restricted to $\eta_{j, k}$ are trivial disk bundles.

Proof. By the result of Vogt [17], List 1, Propositions 5 and 6, $\left(U\left(L_{j, k}\right)\right.$, $F)$ is represented by the vector $(v, 1, u, 1, \cdots, 1)$ or $\left(v, u^{l_{j, k} / 2}, u, 1, \cdots, 1\right)$ (see [17] for details). We may assume that $p_{j, k}\left(\alpha_{1}\right), p_{j, k}\left(\bar{\alpha}_{2}\right) p_{j, k}\left(\bar{\beta}_{2}\right), \lambda$ and $\mu$ are represented by $\left[\overline{\bar{\alpha}}_{l}\right],\left[\overline{\bar{\beta}}_{l}\right](i=1,2,3,4)$ where $\left[\overline{\bar{\alpha}}_{l}\right],\left[\overline{\bar{\beta}}_{l}\right]\left(l=1,2, \cdots, g\left(L_{j, k}\right)\right)$ form a canonical symplectic basis for $H_{1}\left(L_{j, k} ; \mathbb{Z}\right)$. We can take the simple closed curves $\overline{\bar{\alpha}}_{5}$ and $\overline{\bar{\beta}}_{5}$ which satisfy 1$)$ and 2$)$ since $g \geq 8 \max \left(l_{j, k} ; 1 \leq j \leq n, 1 \leq k \leq m_{j}\right)$ +1 implies $g\left(L_{j, k}\right) \geq 5$. If $U\left(L_{j, k}\right)$ restricted to $\overline{\bar{\alpha}}_{5}$ and $\overline{\bar{\beta}}_{5}$ are non-trivial respectively, there is a simple closed curve $\overline{\bar{\eta}}$ on $L_{j, k}$ such that $U\left(L_{j, k}\right)$ restricted to $\overline{\bar{\eta}}$ is trivial and $[\overline{\bar{\eta}}]=\left[\overline{\bar{\alpha}}_{5}\right]+\left[\overline{\bar{\beta}}_{5}\right]$. We put $\eta_{j, k}=\overline{\bar{\eta}}$.

Now we continue the proof of Theorem 23. We perturb $F_{2}$ on $U\left(L_{j, k}\right)$ using $\eta_{j, k}$ in the usual way to obtain a foliation $F_{3}$ which has no compact leaves on the $\pi^{-1}\left(B-\left\{p_{1}, \cdots, p_{s}\right\}\right)$. It is easy to perturb the compact leaves $L_{p_{i}}=\pi^{-1}\left(p_{i}\right)$ $(i=1, \cdots, s)$. This completes the proof.

\section{D) General case.}

Combining Theorems 21, 22 and 23, we have the following.

Theorem 25. Let $F$ be a foliation of a closed 4-manifold $M$ by closed orientable surfaces and $B=M / F$ the leaf space. Suppose $F$ has $m$ rotation leaves with holonomy groups $\mathbb{Z}_{k_{i}}(i=1,2, \cdots, m)$ and $m_{j}$ dihedral leaves with holonomy groups $\mathbb{D}_{l_{j, k}}\left(k=1,2, \cdots, m_{j}\right)$ which correspond to points of $\partial_{j} B$ for each $j(1 \leq$ $\left.j \leq n^{\prime}\right)$.

If $g \geq \max \left(3 \max \left(k_{i} ; 1 \leq i \leq m\right)+1,8 \max \left(l_{j, k} ; 1 \leq j \leq n^{\prime}, 1 \leq k \leq m_{j}\right)+1,7 \varepsilon\right)$, then $F$ is $C^{r}$-unstable $(r \geq 0)$, where $\varepsilon=0$ or 1 and $F$ has no reflection leaves if 
and only if $\varepsilon=0$.

Remark 26. If $g \geq 2$ and $g$ is even, then $F$ has only rotation leaves as singular leaf. Hence Theorem 25 reduces to Theorem 21.

\section{References}

[1] Edwards, R., Millett K. and Sullivan, D., Foliations with all leaves compact, Topology, 16 (1977), 13-32.

[2] Epstein, D., Periodic flows on three-manifolds, Ann. of Math., 95 (1976), 68-82.

[3] —, Foliations with all leaves compaci, Ann. Insi. Fourier, Grenoble, 26 (1976), 265-282.

[4] Fukui, K., Perturbations of compact foliations, Proc. Japan Acad., 5\& Ser. A (1982), $341-344$.

[5] Stability of foliations of 3-manifolds by circles, to appear in J.M.S. Japan.

[6] Fuller, F., An index of fixed point type for periodic orbits, Amer. J. of Math., 89 (1967), 133-148.

[7] Hirsch, M., Stability of compact leaves of foliations, Dynamical Systems, Acad. Press, (1971), 135-155.

[ 8 ] Langevin R. and Rosenberg, H., On stability of compact leaves and fibrations, Topology, 16 (1977), 107-112.

[9] _ - Integral perturbations of fibrations and a theorem of Seifert, Differential topology, foliations and Gelfand-Fuks cohomology, Lecture notes in Math., 652 (1978), 122-127.

[10] Plante, J., Stability of codimension one foliations by compact leaves, Topology, 22 (1983), 173-177.

[11] Reeb, G., Sur certaines propriétés topologiques des varietés feuilletées, Act. Sc. ct. Ind., 1183, Herman, Paris, 1952.

[12] Satake, I., The Gauss-Bonnet theorem for $V$-manifolds, J. M. S. Japan, 9-4 (1957), 464-492.

[13] Seifert, H., Closed integral curves in 3-spaces and isotopic two dimensional deformations, Proc. A. M. S., $\mathbb{1}(1950), 287-302$.

[14] Siegel, C.L., Note on differential equations on the torus, Ann. of Math., \$6 (1945), 423-4.28.

[15] Stowe, D., The stationary set of a group action, Proc. A. M. S., 79 (1980), 139-146.

[16] Thurston, W., A generalization of the Reeb stahility theorem, Topology, $\mathbb{1 3}$ (1974), 347-352.

[17] Vogt, E., Stable foliations of 4-manifolds by closed surfaces, Inv. Math., 22 (1973), 321-348. 
